\title{
Alcance de la información societaria, durante la pandemia del COVID-19.
}

\author{
DOI: $\underline{\text { https://doi.org/10.33262/ap.v3i3.72 }}$
}

Scope of corporate information, during the COVID-19 pandemic

Edison Becerra Molina. ${ }^{1}$, Pedro Astudillo Arias. ${ }^{2} \&$ Yolanda Jaramillo Calle. ${ }^{3}$

\begin{abstract}
.
Introduction. The corporate sector is the outline of commercial companies, national or foreign, that carry out profitable activities, within the current legal and regulatory framework. Objective. Analyze the corporate provisions for the proper management of information, given the uncertainty of COVID-19 in the region 6. Methodology. The design started from the positivist research paradigm, with a non-experimental and crosssectional field design and documentary support, descriptive level, the population was 77,289 and the sample of 471, the questionnaire instrument was used, it consisted of 6 items validated by the trial of three experts with experience in the area of corporate and methodology, qualifying clarity, validity, coherence and relevance. Results. Results with greater frequency with respect to the economic reactivation (26.54\%) consider the injection of capital through loans, (14.52\%) technological updating of equipment, with a systemic vision, using systems and equipment with more advanced technology to stay in the market, ( $13.17 \%$ ) training in digital media such as social networks, new commercial agreements for the reactivation of their companies $(10.34 \%)$, while $(8.46 \%)$ highlights the tariff reduction, $(6.37 \%)$ indicates that the fiscal aid in tax matters would help reactivation, $(5.33 \%)$ prefer market diversification, $(4.60 \%)$ agree that platforms to

\footnotetext{
1 Universidad Católica de Cuenca, Carrera de Contabilidad y auditoría, provincia Azuay, código, jbecerram@ucacue.edu.ec, Orcid.Org ID:0000-0002-6397-9493

${ }_{2}$ Universidad Católica de Cuenca, Carrera de Contabilidad y auditoría, provincia Azuay, código, pastudillo@ucacue.edu.ec, Orcid.Org ID:0000-0003-3088-8322

${ }^{3}$ Universidad Católica de Cuenca, Carrera de Contabilidad y auditoría, provincia Azuay, código, cjaramillo@ucacue.edu.ec, Orcid.Org ID:0000-0003-4639-0318
} 
manage orders and deliveries, (4.28\%) approve communication campaigns, $(3.34 \%)$ prefer the provision of merchandise and inputs for production and $(3.03 \%)$ opt for home delivery of their products or services considering the current situation. Conclution. MSMEs play a relevant role in the economy, due to their potential to generate employability, due to their contribution with the payment of taxes and taxes to the state, as well as contributing to reduce the concentration of economic power and due to their participation in the reactivation and development of the country.

Keywords: COVID-19, Companies, Superintendency of companies, Resolutions, Development.

\section{Resumen.}

Introducción. El sector societario es el contorno de las empresas mercantiles, nacionales o extranjeras, que desarrollan actividades rentables, dentro del marco legal y normativo vigente. Objetivo. Analizar las disposiciones societarias para el manejo apropiado de la información, ante la incertidumbre del COVID-19 en la región 6. Metodología. El diseño partió del paradigma de investigación positivista, con diseño de campo no experimental y transversal y apoyo documental, nivel descriptiva, la población fue de 77.289 y la muestra de 471, se utilizó el instrumento del cuestionario, constó con 6 items validados por el juicio de tres expertos con experiencia en el área de societaria y metodología, calificando claridad, validez, coherencia y pertinencia. Resultados. Resultados con mayor frecuencia con respecto a la reactivación económica $(26.54 \%)$ consideran la inyección de capital mediante préstamos, (14.52\%) actualización tecnológica de equipos, con visión sistémica, utilizando sistemas y equipos con tecnología más avanzada para mantenerse en mercado, (13.17\%) capacitación en medios de comunicación digitales como redes sociales, acuerdos comerciales nuevos para la reactivación de sus empresas $(10.34 \%)$, mientras $(8.46 \%)$ resalta la reducción arancelaria, $(6.37 \%)$ señala que la ayuda fiscal en tema de impuestos permitiría la reactivación, (5.33\%) prefieren la diversificación de mercado, $(4.60 \%)$ concuerdan que las plataformas para gestionar pedidos y entregas, $(4,28 \%)$ aprueban las campañas comunicacionales, (3.34\%) prefieren la provisión de mercadería e insumos para la producción y un (3.03\%) optan por la distribución a domicilio de sus productos o servicios considerando la situación actual. Conclusión. Las Mipymes cumplen un rol relevante en la economía, por su potencial de generar empleabilidad, por su aporte con el pago de tributos e impuestos al estado, asi como contribuir a reducir la concentración de poder económico y por su participación en la reactivación y desarrollo del país.

Palabras claves: COVID-19, Compañías, Superintendencia de compañías, Resoluciones, Desarrollo. 


\section{Introducción.}

La crisis económica generada por la enfermedad del coronavirus (COVID-19), ha tenido un impacto importante a nivel local, regional, nacional y mundial ha golpeado la estructura productiva y empresarial con extenuaciones que se han originado a lo largo de décadas. El fenómeno asociativo tiene en la actualidad mucha relevancia, de tal forma que las personas naturales como jurídicas se reúnen para alcanzar los más diversos fines empresariales, de allí que las personas se agrupan con propósitos económicos, empresariales, políticos, religiosas, culturales, deportivos, entre otros.

El sector societario en nuestro país el Ecuador, lo constituyen las sociedades o empresas mercantiles, nacionales o extranjeras, las asociaciones o consorcios que las empresas formen, que se encuentran bajo control de la Superintendencia de Compañías, Valores y Seguros, mismas que desarrollan actividades beneficiosas, considerando el marco legal y normativo vigente.

En el setor societario participan personas naturales o jurídicas que ostentan la calidad de accionistas, socios, administradores, liquidadores, comisarios, auditores, interventores, dichas actuaciones están reglamentadas por la Ley de Compañías y la respectiva normativa secundaria o resoluciones.

Las compañías tiene trascendental importante, principalmente para el desarrollo y crecimiento del país, porque tanto las micro, pequeñas y medianas empresas estudiadas generan empleabilidad, impuestos y patrimonio, mediante los servicios que prestan, asi como de los bienes que produce, que permite satisfacer las necesidades de los consumidores o usuarios, asi como satisface los intereses de los accionistas, socios, proveedores, acreedores, empleados, y demás actores, es decir benefician a la humanidad en su conjunto.

La presente investigación es parte del proyecto de investigación denominado "La importancia de la información contable y financiera ante la incertidumbre del COVID-19 en las Mipymes en el entorno de los negocios en la región 6", el mismo que se justifica porque contribuyó desde una perspectiva teórica académica a dar salida a los problemas de las compañias, desde el punto de vista del contenido societario y contable, estos cambios se realizaron para apoyar a las empresas en ese momento de paralización económica que sufrió el país, a partir de la presencia del coronavirus (COVID-19).

\section{Metodologia.}

Sobre la base de nuestro investigación se detalla cómo se sistematiza el trayecto metodológico que se utilizó para llevar a cabo el estudio, parte del paradigma de investigación positivista, con diseño de campo no experimental y transversal con apoyo documental, el nivel de la investigación fue la descriptiva, al respecto Hurtado (2010), explica que su propósito es exponer el evento estudiado, haciendo una enumeración 
detallada de sus características, de modo tal que en los resultados se pueden obtener dos niveles de análisis, dependiendo del fenómeno y del propósito del investigador, es un nivel más elemental, en el cual se logra una clasificación de la información en función de las características comunes, y un nivel más sofisticado en el cual se ponen en relación los elementos observados" (p. 73). En consecuencia se corresponde con el nivel descriptivo en función del objetivo general planteado, la población estuvo estructurada por tres sectores escogidos, microempresas, considerando la base del catastro del SRI, del 2021, así Azuay con 45.814, Cañar 10.497 y Morona Santiago 11.300, total 67.611 microempresas de la Zona 6 del Austro, Empresas registradas en la Superintendencia de Compañías por el año 2021, Azuay 8.843, Cañar 494 y Morona Santiago 341, total 9.678, dando un gran total de 77.289, de cual se determinó el tamaño de la muestra con un 4,50\% de error, correspondiendo la muestra $n=471$, mismas que fueron aplicadas mediante la herramienta Google forms, la validez de contenido fue realizado mediante el juicio de tres expertos con experiencia en el área de compañias, contabilidad y metodología.

En tal sentido, fue importante analizar en primer lugar, la pertinencia del coeficiente de consistencia interna de Cronbach, el misma habla, sobre el grado en que los ítems miden la homogeneidad.

De la misma manera, para establecer la confiabilidad del cuestionario elaborado, se procedió aplicar una prueba piloto a 30 empresas, que no forman parte de la muestra, todos ellos con características similares a los sujetos de este estudio, administrando una escala, de 30 ítems, a una muestra de $\mathrm{n}=30$. De acuerdo con los resultados se concluyó que la escala tiene una confiabilidad de consistencia interna muy alta, suficiente como para ser considerada fiable. En conclusión, la confiabilidad del instrumento fue de 0,9 muy alta, así mismo, fue importante la utilización de la herramienta estadística para el desarrollo de la investigación, recurriéndose a la estadística descriptiva, lo que permitió la recolección, organización y presentación de datos, facilitando, con el apoyo de cuadros, tablas y figuras, los resultados obtenidos de la aplicación de los cuestionarios en relación con los objetivos planteados del presente estudio, mediante el software libre SPSS, versión 26.

Con respecto, al procesamiento y análisis de los datos obtenidos a lo largo del proceso de investigación, se realizó, considerando el uso de la triangulación, validez, confiabilidad y contrastación de los instrumentos.

\section{Fundamentación Teórica.}

La pandemia de COVID-19 ha causado una contracción de la actividad económica sin precedentes en todo el mundo, y nuestra región y país no es una excepción, los efectos causados a la economía son destructores, es el peor, desde la segunda guerra mundial, según, El Fondo Monetario internacional -FMI (2020). Esta evolución ha tenido un impacto negativo en las micro, pequeñas y medianas empresas, que están sufriendo fuertes caídas de la rentabilidad y un deterioro de su capacidad de repago. 
Por otro lado, la Organización Mundial de la Salud -OMS (2021), declara pandemia mundial por la expansión del SARS-CoV-2, coronavirus causante de la enfermedad COVID-19. Siete meses después, el virus ha causado sobre 40 millones de contagios a nivel mundial y más de 1 millón de fallecidos. Las medidas adoptadas por los distintos gobiernos, tales como cese temporal de actividades, reducción de desplazamientos, cierre de comercio y servicios, entre otras, han generado un grave impacto en las economías locales y en el empleo.

Según, la Comisión Económica para América Latina y el Caribe -CEPAL (2021). La pandemia del COVID-19 está produciendo un impacto muy profundo sobre la economía y la sociedad de América Latina y el Caribe. La CEPAL estimó que hasta finales del año 2020 cerraron 2,7 millones de empresas, equivalentes al 19\% de todas las empresas de la región. En el caso de las microempresas este porcentaje llegó al 21\%. En términos de empleo, esto generó la destrucción de más de 8,5 millones de puestos de trabajo: $8,1 \%$ del total del empleo formal en el sector empresarial y más de un quinto de los puestos de trabajo generados por el sector de las microempresas.

Los datos de los mercados financieros muestran que el mayor impacto lo sufren las micro, pequeñas y medianas empresas (Mipyme), mismas que se han visto más perjudicadas por el shock de la COVID-19, con caídas de sus ventas, no cuentan con grandes colchones de liquidez y se enfrentan a condiciones de financiamiento.

En nuestro país, y de manera específica en la región zona 6, surge la necesidad de reprogramar y reorganizar el desarrollo productivo de las pequeñas y medianas empresas, con protocolos que permitan volver paulatinamente a la normalidad, para esto existen convenios entre entidades públicas y privadas que van configurando la nueva manera de reiniciar el trabajo en cada una de las Mipymes, con actividades comerciales, para poder enfrentar tan desfavorable situación en correcta relación con las medidas sanitarias que impidan una ola de contagio no deseada, se vienen impulsando muchas maneras de reactivar la producción y la economía, como el teletrabajo, el trabajo por turnos, en fin una serie de estrategias innovadoras para salir de esta situación problemática.

Por su parte, la Ley Orgánica de apoyo humanitario (2020), para combatir la crisis sanitaria derivada del COVID-19, tiene por objeto establecer medidas de apoyo humanitario, necesarias para enfrentar las consecuencias derivadas de la crisis sanitaria ocasionada por el COVID-19, a través de medidas tendientes a mitigar sus efectos adversos dentro del territorio ecuatoriano; que permitan fomentar la reactivación económica y productiva del Ecuador, con especial énfasis en el ser humano, la contención y reactivación de las economías familiares, empresariales, la popular y solidaria, y en el mantenimiento de las condiciones de empleo (p.6).

En cuanto al portento asociativo aparece justificado por la carta magna que rige el Ecuador, es asi que la constitución en el Capítulo VI, que se corresponde con el derechos de libertad, en su Art. 66, numeral 15 el cual manifiesta: "El derecho a desarrollar actividades económicas, en forma individual o colectiva, conforme a los principios de 
solidaridad, responsabilidad social y ambiental", reconociendo así, el derecho a la libre asociación para alcanzar un fin común.

Por su parte, el Código Civil (2005), en su Art. 1957 define a la sociedad o compañía como el "contrato en el cual dos o más personas estipulan poner algo en común (dinero, bienes servicios, industria o trabajo apreciables en dinero), con el fin de dividir entre sí los beneficios que de ello provengan".

Es conveniente destacar que el desarrollo de la sociedad, constituye el un eje $\mathrm{N}^{\circ} 2$ del Plan Nacional de Desarrollo 2017-2021 "Toda una Vida" (2021), "Economía al Servicio de la Sociedad", en cuanto a este eje se toma como punto de partida la premisa de que la economía debe estar al servicio de la sociedad. Es así que nuestro sistema económico, por definición constitucional, es una economía social y solidaria. Dentro de este sistema económico interactúan los subsistemas de la economía pública, privada, popular y solidaria. No se puede entender la economía sin abarcar las relaciones entre los actores económicos de estos subsistemas, que son de gran importancia y requieren incentivos, regulaciones y políticas que promuevan la productividad y la competitividad.

La Ley de Compañías (2014) es el ordenamiento jurídico principal que regula a las sociedades mercantiles y cuya normativa define a la compañía como el "contrato mediante el cual dos o más personas unen sus capitales o industrias para emprender en operaciones mercantiles y participar de sus utilidades".

En el Ecuador, la entidad o institución encargada del Control y Vigilancia de la Compañías, es la Superintendencia de Compañías, Valores y Seguros, entidad que por mandato constitucional y legal controla las sociedades anónimas, compañías de responsabilidad limitada, de economía mixta, compañías en comandita por acciones y las nuevas sociedades por acciones simplificadas, además es quien dicta los reglamentos para la aplicación tanto de la Ley de Compañías, así como los acuerdos de las partes derivadas del contrato o convención mercantil; la compañía en nombre colectivo y la de comandita simple corresponde su aprobación al Juez de lo Civil del Consejo de la Judicatura del domicilio en que se constituyan.

Hay seis variedades de compañías de comercio, entre las que tenemos: La compañía en nombre colectivo, la compañía en comandita simple y dividida por acciones, la compañía de responsabilidad limitada, la compañía anónima, la compañía de economía mixta; y las sociedades simplificadas por acciones que vienen creciendo de manera vertiginosa, por tales razones las Mipymes en Ecuador y específicamente el la región 6, son fundamentales en la economía, se han convertido en una fuente de generación de empleo y un medio para ofrecer productos y servicios diversos en mercados de menor tamaño.

\section{Resultados.}

Los resultados, constituyen hallazgos derivados del presente estudio, en correspondencia con la finalidad de la investigación, con el objeto de articular los efectos de la pandemia 
ante disposiciones emanadas por el gobierno central con las sociedades, reguladas por la Superintendencia de Compañias, Valores y Seguros para intentar no solamente conocer su impacto, si no dar respuesta a los entornos locales en relación con las Mipymes.

La tabulación e interpretación de los cuestionarios dirigidos a las micro, pequeñas y medians empresas de la región 6 , fueron un total de 10 ítems, para el presente análisis se han seleccionado únicamente seis, los más notables.

Figura 1. Clases de empresas.

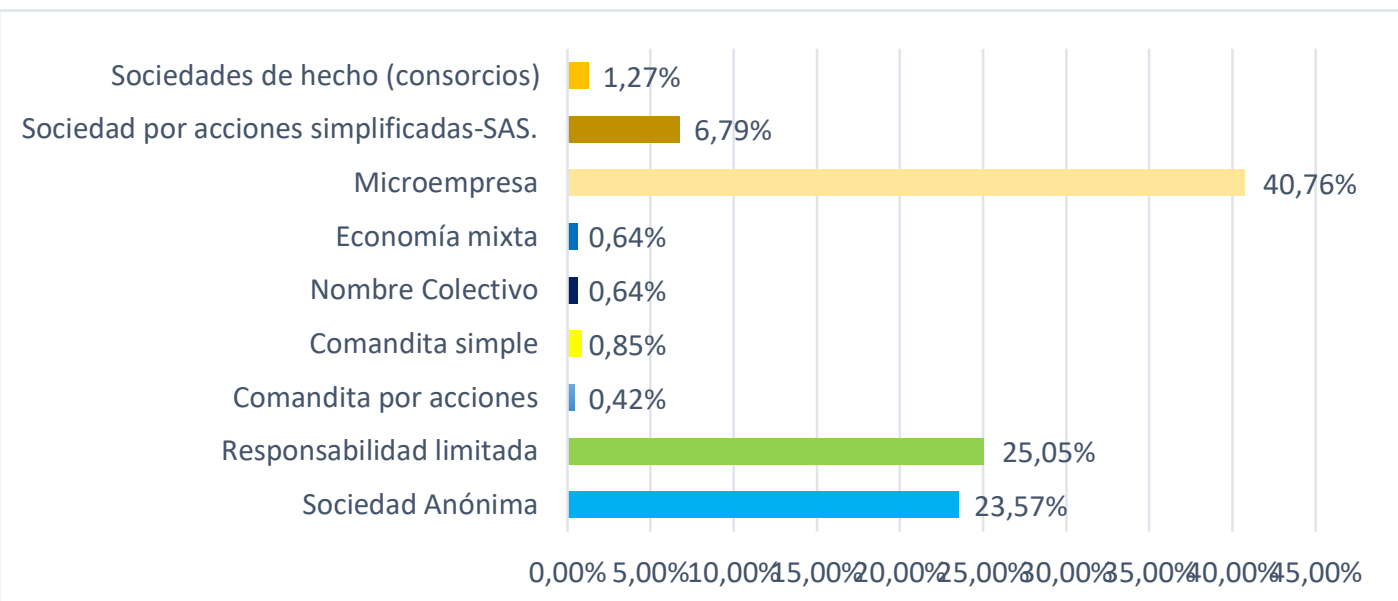

Fuente: Elaboración propia (2021).

De acuerdo a las encuestas realizadas podemos observar que las clases de empresas que existen en nuestro ámbito societario, tiene diferentes porcentajes dando los siguientes resultados: Comandita por Acciones; $0,42 \%$, se caracterizan por cuanto no requiere capital mínimo para su constitución, cada socio participa directamente en los beneficios, la posibilidad de reunir un mayor capital para la empresa, las responsabilidades ante las obligaciones sociales de la empresa son establecidas según el tipo de socios comanditarios o comanditados; Economía Mixta 0,64\%, se definen por que son empresas dedicadas al desarrollo y fomento de la agricultura, industria, prestación de servicios públicos, aporte económico del sector público y privado, sector privado participa en el capital y gestión social; Nombre Colectivo; 0,64\%, se determinan por la posibilidad de reunir un mayor capital para la empresa, todos los socios responden de manera subsidiaria, solidaria e ilimitadamente a las obligaciones de la Sociedad, cada socio participa directamente en los beneficios, cada socio tiene derecho a un voto salvo pactado en lo contrario; Comandita Simple $0.85 \%$, es una sociedad mercantil personalista, con razón social y capital social representado por partes sociales nominativas; suscritas por uno o más socios comanditados; Sociedad de Hecho (consorcios); 1,27\%, es decir corresponden a un consorcio o asociación económica en la que una serie de empresas buscan desarrollar una actividad conjunta mediante la creación de una nueva sociedad; Sociedad por Acciones Simplificadas- SAS.; 6,79\%, este tipo de empresas constituyen la transformación más importante del Derecho Societario Ecuatoriano, cuyo objetivo principal es facilitar al emprendedor en la realización de sus actividades mercantiles, permite reducir costo y 
tiempo, los socios solo serán responsables hasta por el monto de sus respectivos aportes. Sociedad Anónima 23,57\%, sus ventajas estan dadas porque se pueden transmitir las acciones mediante su venta, los acreedores tienen derecho sobre los activos de la compañía, no sobre los bienes de los accionistas, el dinero que los accionistas arriesgan al invertir en una Sociedad Anónima se limita al valor de su inversión, obtención de crédito, cada socio participa directamente en los beneficios; Responsabilidad Limitada $25,05 \%$, su importancia radica en el hecho de que no demanda capital mínimo para su constitución, cada socio participa directamente en los beneficios, la posibilidad de reunir un mayor capital para la empresa; y Microempresa $40,76 \%$. Se caracerizan por poseer entre 1 y 9 trabajadores en promedio. Esto es así, independientemente de que el negocio se dedique a la industria, comercio o los servicios. Podemos ver entonces que cada empresa está formada según a su función que realiza y apegada a los reglamentos que la ley dispone de igual forma cada una de ellos son entes económicos que generan trabajo y sostenibilidad dentro de la sociedad por más mínimo que sea su aporte en la economía, de allí que son de gran ayuda para un país en desarrollo y que se encuentra impulsando la reactivación económica ante la presencia de la pandemia del COVID-19.

Figura 2. Importante de constitución legal.

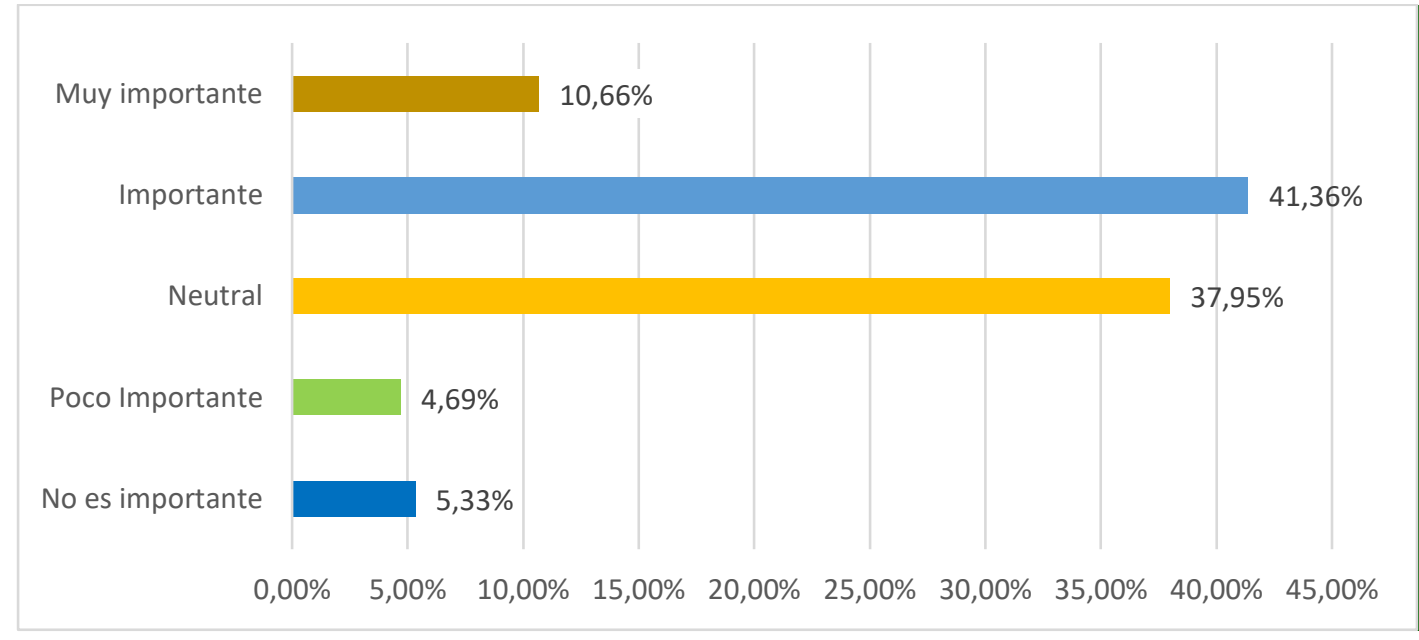

Fuente: Elaboración propia (2021).

El analisis, nos permiten conocer la importancia que se le da a la constitución legal de una empresa para emprender en la etapa de recesión causada por el COVID-19, el $41.36 \%$, el criterio es muy importante el $10.66 \%$, al unir al tendencias representa el $52.02 \%$, mantiene un criterio neutral $37,95 \%$, el $5.33 \%$ considera que no es importante y un $4.69 \%$ considera que es poco importante. La elección de constituirse en forma jurídica de una empresa es esencial, ya sea como persona física, sociedad mercantil, cooperativa, entre otros, esta elección es la que nos va a posicionar dentro del mercado, dentro del negocio de internet y las redes sociales, además de ser un elemento importante en la fiscalidad, sujeto de crédito, posibilidades de captación de clientes, y que la misma sea capaz de producir, comercializar y promocionar los productos con autoridad y sin restricciones. 
Figura 3. Medidas de las empresas ante la presencia Covid-19

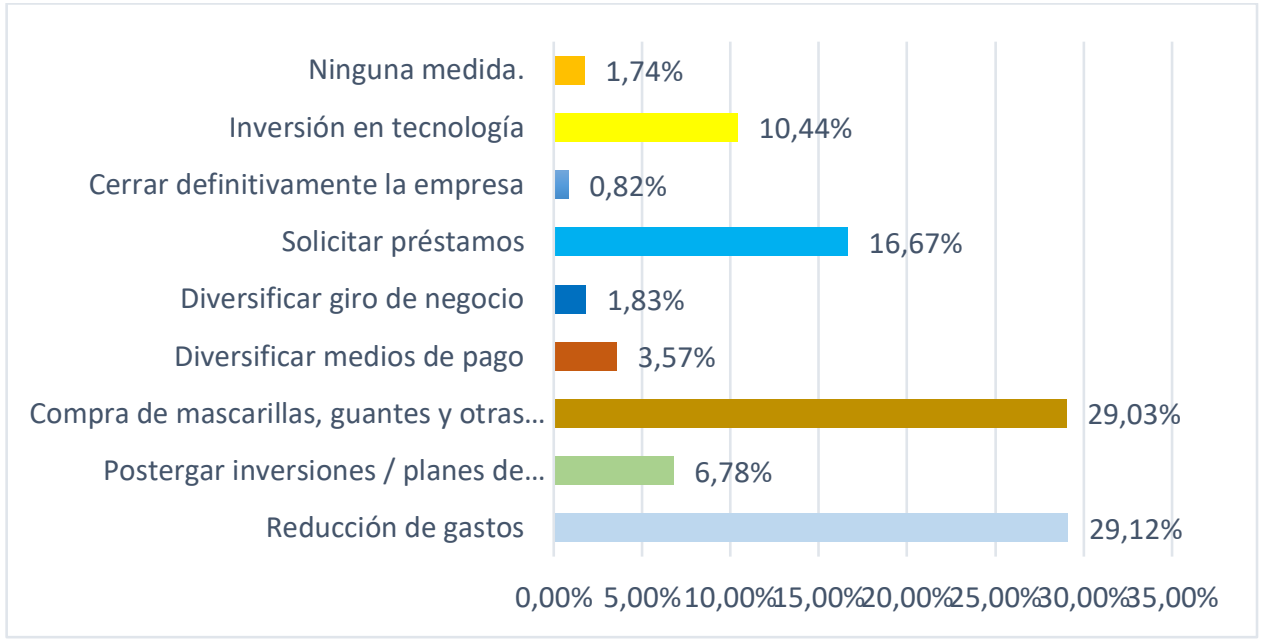

Fuente: Elaboración propia (2021).

La información recogida permitió determinar que las medidas que se han llevado a cabo ante la crisis de la pandemia del Covid-19, un 29,12\% ha optado por la reducción de gastos, se ha implementado este tipo de medida para impedir el cierre de la empresa, pero tampoco tienen el presupuesto necesario para cubrir obligaciones corrientes. Por otra parte, el 29,03\% ha realizado la compra de mascarillas, guantes y otros servicios de limpieza, hay que resaltar que Ecuador no es el único país que ha optado por esta medida, debido al COVID-19 y a la crisis económica, muchos empresarios y emprendedores, han optado por seguir adelante en el mercado cuidando a sus empleados con medidas de bioseguridad.

Asi mismo, dentro de las medidas apoptadas un 16,67\% han preferido solicitar préstamos a las instituciones financieras para cubir sus obligaciones inmediatas, debido a la crisis sanitaria y económica, muchas de las empresas han quebrado, sin embargo, han considerado en mantener su empresa y no cerrarla, ante la presenica de la pandemia del COVID-19. De igual forma se han realizado inversiones en tecnología el 10,44\%; por que consideran que facilita las labores comerciales, mediante las computadoras y las tablets, combinadas con una señal de internet, por ejemplo, permiten un procesamiento más rápido y sencillo de los productos comercializados también podrían fomentar el comercio de bienes sensibles al tiempo actual, por ello la tecnología permite desarrollar un enorme gama de servicios, mercados más amplios y nuevas formas de generar negocios, los procesos tecnológicos hay que saberlos producir, diseñar y crear, una empresa que utiliza tecnología actual es menos propensa a presentar un declive.

Asi mismo, el 6,78\% en postergar inversiones/ planes de crecimiento para su empresa, esto es debido a la crisis economía y financiera que se ha dado a causa de la pandemia del COVID-19, con un 3,57\% en diversificar medios de pago, hoy en día vivimos en un mundo cada vez más interconectados, empujados a la tendencia de digitalización, por ello los métodos de pago se realizan mediante las transacciones electrónicas mediante tarjetas de debito y crédito. 
De igual forma el 1,83\% diversificar giro de negocio, radica en crear nuevos modelos de negocio, depositar el negocio en distintas vías de manera que si una falla las restantes pueden dar resultados y si todas funcionan existe más beneficios para la empresa. El $1,74 \%$ no tomo ninguna medida y por último el $0,82 \%$ sea llegado a la decisión del cierre definitivo de empresa. En medida de ello las diferentes estrategias asumidas por las empresas para enfrentar el periodo de pandemia, y con ello poder sobrellevar la crisis existente. La estrategia de las empresas consiste en crear un plan de acción que considere la situación actual que tiene una empresa o negocio y hacia donde quieren y pueden llegar, por las experiencias de quien tiene el papel de dirigir las empresas, para tener o mantener una ventaja competitiva.

Figura 4. Riesgo por la presencia del COVID 19.

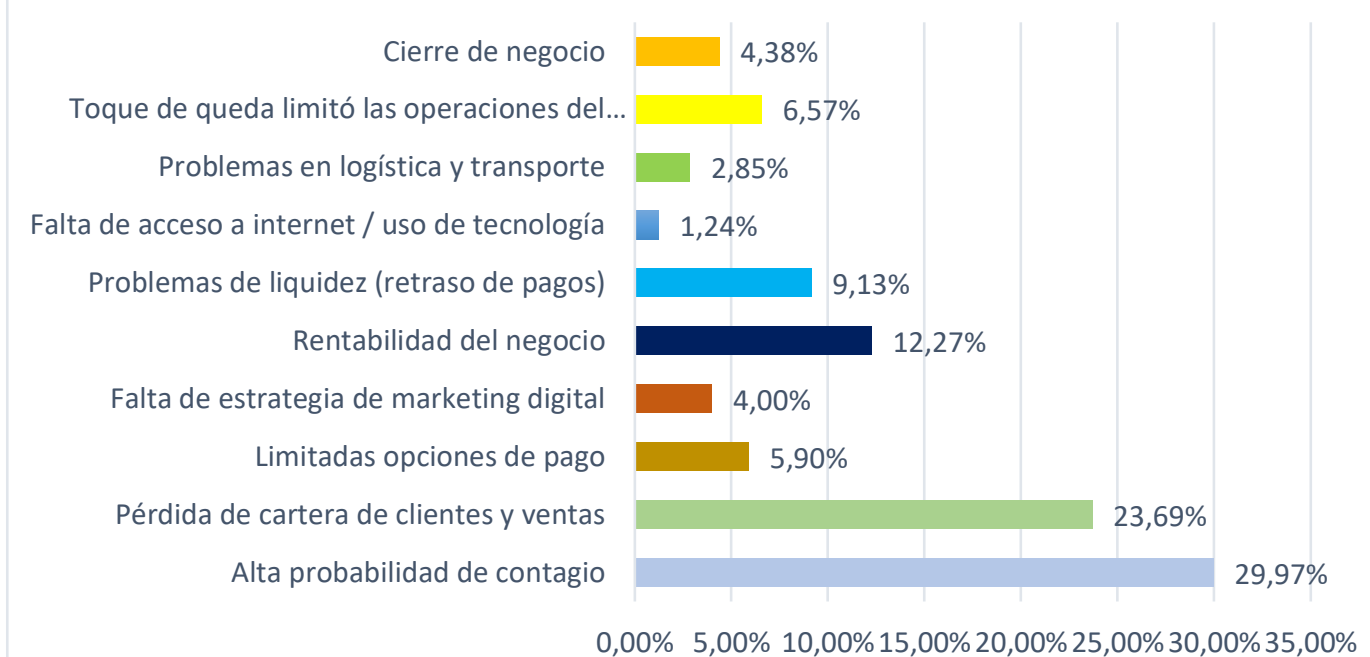

Fuente: Elaboración propia (2021).

De igual manera, la figura nos muestra que la preocupación que más alerto a los encuestados o empresarios debido a la presencia del COVID 19 es la alta probabilidad de contagio que existe al realizar sus actividades con un $29,97 \%$, esto es comprensible ya que las personas tratamos de velar por nuestra salud. Del mismo modo otras preocupaciones son la pérdida de cartera de clientes y ventas que los negocios que pueden tener con un $23,69 \%$, esto es el resultado de la pérdida de valor de determinados montos que las empresas tiene pendientes de reclamar a sus clientes por los bienes o servicios entregados o vendidos, por otra parte los consumidores al tener temor de un contagio estas no realizan el consumo, la rentabilidad de los negocios también se cree que serán afectados por la actual situación con un $12.27 \%$ ya que no solo afecta a ciertos sectores en específico sino esto afecta a todo el mercado y como consecuencia tenemos que la rentabilidad de estos no sean prósperos. Igualmente los problemas de la liquidez (retraso de pagos) que pueden tener con sus proveedores, empleados, la seguridad social, la administración tributaria, entre otros, en el 9.13\%, al tener menor afluencia de consumidores las ventas y producción no ayudarían con la cancelación de estos. 
En cuanto al toque de queda limitó las operaciones del negocio con un 6,57\%, consiste en limitar la libertad de movimiento de los ciudadanos durante una franja horaria, como fue en la noche, o en días determinados. En relación a las limitadas opciones de pago ocupan el $5,90 \%$, porque no todos poseemos una cuenta bancaria para las transferencias o bien no se tiene una tarjeta de crédito, el cierre de negocios cuenta con un $4.38 \%$ al no tener la misma clientela muchas de las veces el producto se queda varado y esto ha forzado al negocio a cerrar, la falta de estrategia de marketing digital también se hace presente con un 4\%, una estrategia de marketing digital consiste en la planificación de ciertos pasos para llegar a objetivos determinados por medios online, que conlleva aspectos como la creación y difusión de contenidos a través de los sitios web y las redes sociales, la gestión de correos electrónicos y blogs.

Por su parte, los problemas de logística y la falta de acceso a internet/uso de tecnología fueron de las opciones menos consideradas ya que cuentan con un $2,85 \%$ y de $1,24 \%$ respectivamente. Cada uno de los problemas descritos formaron parte de la realidad actual de las empresas y con la consecuente aparición de nuevas variantes como la alfa y la delta, variantes del coronavirus que se ha encontrado en más de 80 países desde que la detectaron en el Reino Unido (Gran Bretaña y la India) en su orden y otras que seguirán asomado seguramente, en síntesis, el real temor es que esto siga o a su vez que resurjan otras problemas, lo cual solo se podrá ver en medida que el tiempo avance.

Figura 5. Opciones para reactivación económica de las empresas

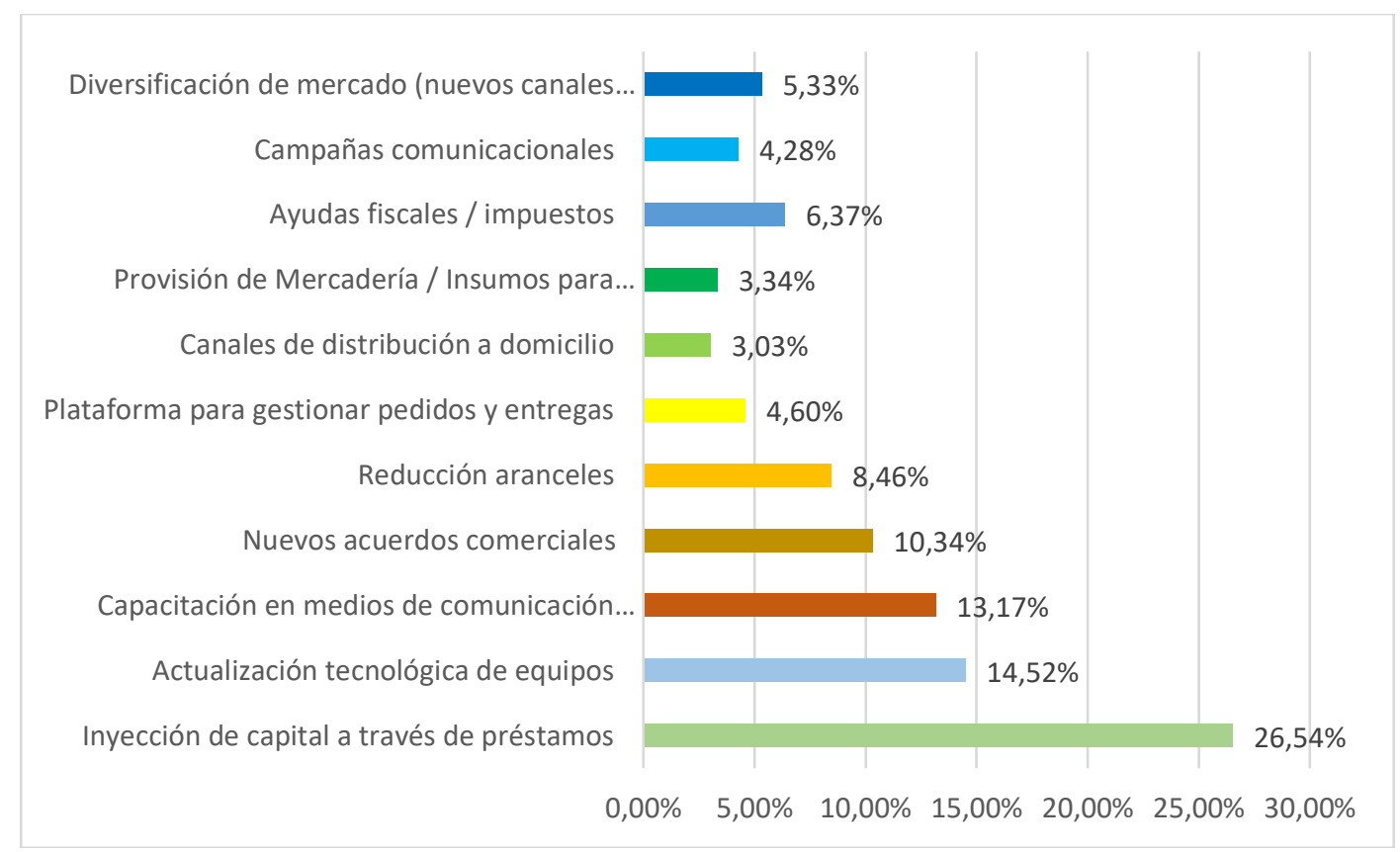

Fuente: Elaboración propia (2021).

Los ejecutivos empresariales en el $26.54 \%$ consideran la inyección de capital a través de préstamos para reactivar la economía de sus empresas, mediante las entidades financieras de capitales que conceden préstamos a empresas nuevas para que puedan impulsar su negocio y tener liquidez para que pueda hacer frente a sus deudas y obligaciones a corto plazo, de allí que los ejecutivos empresariales deben tener en cuenta la liquidez de la 
empresa a la hora de tomar decisiones financieras, que podría ser para posibles inversores en la misma. Asi mismo el $14.52 \%$ la actualización tecnológica de equipos; por consiguiente, comprende un conjunto de acciones, con visión sistémica, con el objetivo de actualizar los equipos y sistemas considerados obsoletos, utilizando medios y equipos con tecnología más avanzada para mantenerse en mercado, con un $13.17 \%$ la capacitación en medios de comunicación digitales como redes sociales, páginas web entre otras.

Por otra parte, del total de los encuestados optan por acuerdos comerciales nuevos para la reactivación de sus empresas con un $10.34 \%$, es decir alianzas comerciales y adopción de canales de pago digitales y oportunidades de financiamiento para impulsar su reactivación. Mientras que el $8.46 \%$ resalta que la reducción arancelaria sería la mejor opción, como parte de la política comercial, que permite generar facilidades a la importación de productos y todos aquellos suministros que son necesarios para su uso y consumo en la economía nacional y de la producción. El $6.37 \%$ señala que la ayuda fiscal en tema de impuestos ayudaría a la reactivación. También se determinó que un 5.33\% de los encuetados prefieren la diversificación de mercado, que es un proceso que se utiliza a menudo por las empresas para mejorar sus posiciones y asegurar un flujo constante de ingresos. Así también el $4.60 \%$ concuerdan que las plataformas para gestionar pedidos y entregas sería lo ideal para la reactivación económica de cada una de sus empresas.

Por otro lado un 4,28\% aprueban las campañas comunicacionales como la mejor manera de reactivación económica de las empresas, mientras que el 3.34\% prefieren la provisión de mercadería e insumos para la producción; y finalmente un 3.03\% optan por la distribución a domicilio de sus productos o servicios considerando la situación actual. Cada una de las alternativas son valederas a la hora de gestionar de una mejor manera las empresas, se ve un alto interés en participar dentro de las estrategias del e-comerce, lo cual son acciones que en el país es algo bastante nuevo, pero que a razón de la situación se ha tenido que implementar, lo que ha creado alternativas de comercio mucho más efectivas y cómodas.

Figura 6. Dificultades para adquirir productos y servicios

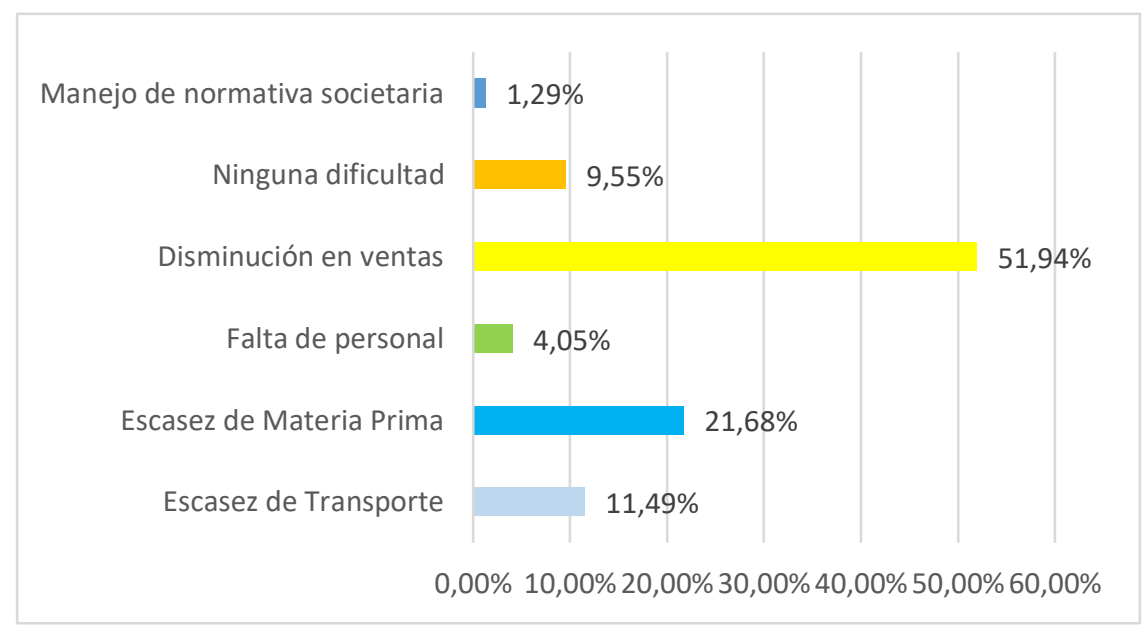

Fuente: Elaboración propia (2021). 
Es conveniente destacar que el 51.94\% de las empresas ha tenido disminución de las ventas, pues las ventas son el elemento principal que permite a la empresa conseguir ingresos, por tanto no podemos dejar a un lado factores que influyen en el crecimiento o decrecimiento de estas, uno de los factores que han incidido es la pandemia del COVID 19. La rentabilidad de una empresa, y su sostenimiento a largo plazo, depende de sus ingresos, y los ingresos dependen de las ventas, por ello es la piedra angular en la superviviencia de las compañías, así mismo el $21.68 \%$ de los encuestados manifiestan haber tenido problemas debido a la escasez de materia prima, mientras que el $11.49 \%$ tuvo problemas con la escasez de transporte, por el contrario, el 9.55\% señala no tener ningún tipo de dificultad con su empresa y finalmente el $1.29 \%$ manejo de normativa, toda vez que especiamente los propietarios de algunas compañias y en particular las sociedades por acciones simplificadas, con respecto a los deberes societario y las tendencias que se observan en el ámbito mercantil, deberán estar debidamente inteligenciados de sus obligaciones y responsabilidades.

\section{Análisis medidas societarias}

La aparición de la pandemia Coronavirus (COVID-19), afectó a las empresas en todo el mundo, obligó a las empresas a suspender sus actividades comerciales, pero el desempeño ha sido muy variable, incluso dentro de un mismo país o región, de acuerdo a nuestra investigación es procedente mencionar algunas acciones en materia societaria desplegadas por el gobierno y el organismo de control, es decir la Superintendencia de Compañías, Valores y Seguros, desde finales del mes de enero del año 2020 hasta inicios del mes de julio del 2021, siendo las principales las siguientes:

La Resolución SCVS-INAF-DNF-2020-003, de fecha 27 de febrero del 2020, con registro Oficial 150, en donde se da a conocer la contribución que las compañías y otras entidades sujetas a la vigilancia y control de la Superintendencia de Compañías, Valores y Seguros deben pagar a ésta, para el año 2020, de conformidad con lo que establece el inciso tercero del artículo 449 de la Ley de Compañías, será de acuerdo con lo especificado en lo siguiente:

Tabla de contribución que deben pagar las compañías y otras entidades

\begin{tabular}{|r|r|r|r|}
\hline $\begin{array}{r}\text { MONTO DEL ACTIVO REAL DE LAS COMPAÑÍAS (EN US } \\
\text { DÓLARES) }\end{array}$ & $\begin{array}{c}\text { CONTRIBUCIÓN POR MIL SOBRE EL ACTIVO } \\
\text { REAL }\end{array}$ \\
\hline \multicolumn{1}{|c|}{ DESDE } & & HASTA & 0,00 \\
\hline $75.000,01$ & - & $15.000,00$ & 0,71 \\
\hline $100.000,01$ & - & $1.000 .000,00$ & 0,76 \\
\hline $1.000 .000,01$ & & $20.000 .000,00$ & 0,82 \\
\hline $20.000 .000,01$ & - & $500.000 .000,00$ & 0,88 \\
\hline $500.000 .000,01$ & - & EN ADELANTE & 0,94 \\
\hline
\end{tabular}

Fuente: Elaboración propia (2021). Basado en la resolución SCVS-INAF-DNF-2020-003 de la Supercias.

Posteriormente, La situación antes planteada dió lugar a determinar una prórroga para presentar estados financieros por parte de la Superintendencia de Compañías, por ello se 
suspendió los plazos y términos de todos los procesos administrativos, coactivos, tributarios y de prescripción de acción de cobro desde el 16 de marzo al 16 de mayo. Además de la prórroga para presentar información del Art. 20, conforme estipula la Ley de Compañias (2014), con respecto a los estados financieros, nomina de socios y/o accionistas, nomina de administradores, estado de flujo del efectivo, estado de cambios en el patrimonio, notas a los estados financieros, informe de gerencia, informe de comisario, informe de auditoria externa y acta de junta general, se extiende hasta 30 de junio del 2020, es de indicar que el plazo anterior no comprende entrega de información por parte de compañías extranjeras del Art. 23 de la Ley de Compañias, conforme Resolución No SCVS-INPAI-2020-0002715.

Por su parte, la contribucion a la Superintendencia de Compañias, cuyo pago de contribución del ejercicio fiscal 2019 a pagarse en 2020, se determinó que debía realizarse de la siguiente forma: a) 50\% a cancelar hasta el 30 de septiembre de 2020; y, b) 50\% a cancelar hasta el 31 de diciembre de 2020, según resolución № SCVS-INAF-DNF-20200007 de fecha 15 de abril del 2020.

Igualmente, según resolución $\mathrm{N}^{\circ}$ SCVS-INPAI-DNAI-2020-00003179, Para los casos concretos en los cuales se deba efectuar ya sea a petición de parte o de oficio, la práctica de una audiencia para garantizar la inmediación en un procedimiento administrativo, estas se realizarán priorizando la utilización de medios telemáticos en las plataformas virtuales que se indiquen en las respectivas providencias de señalamiento de fecha y hora para la videoaudiencia, en las mismas se proporcionarán de ser el caso, los correspondientes códigos o contraseñas para ingresar a las mencionadas plataformas.

De igual forma, la resolución No SCVS-INPAI-2020-00003532, de fecha 17 de junio del 2020, se procede a ampliar la suspensión de los plazos y términos prevista en el artículo uno de la resolución $\mathrm{N}^{\circ}$ SCVS-INPAI-2020-00003186 de 15 de mayo de 2020, referente a todos los procesos administrativos, coactivos, tributarios y de prescripción de la acción de cobro, y en general de todo proceso cuya sustanciación sea inherente a las competencias de la Superintendencia de Compañías, Valores y Seguros, que se encuentren discurriendo en este ente de control, contada a partir del 19 de junio de 2020.

Del mismo modo se dispone que los plazos y términos de todos los procesos administrativos, coactivos, tributarios, de prescripción de la acción de cobro y en general de todo proceso cuya sustanciación fuere inherente a las competencias de la Superintendencia de Compañías, Valores y Seguros, que se encontraban suspendidos por efectos de la aplicación de las resoluciones números: SCVS-INPAI-2020-00002712, de 16 de marzo de 2020; SCVS-INPAI-2020-00002946, de 16 de abril de 2020; SCVSINPAI-2020-00003186, de 15 de mayo de 2020; y, SCVS-INPAI-2020-00003532, de 17 de junio de 2020; vuelvan a discurrir a partir del 02 de julio de 2020, resolución de fecha 29 de junio del 2019, № SCVS-INPAI-2020-00003735.

Asi mismo, la resolución $\mathrm{N}^{\circ}$ SCVS-INC-DNCDN-2020-0015, de fecha 14 de septiembre del 2020, expide el EXPEDIR EL REGLAMENTO DE LAS SOCIEDADES POR 
ACCIONES SIMPLIFICADAS (S.A.S.), puesto que las sociedades por acciones simplificadas, una vez constituida, tiene personalidad jurídica independiente de sus accionistas, el principio de existencia jurídica de este tipo de sociedades es la fecha de inscripción del documento constitutivo en el Registro de Sociedades de la Superintendencia de Compañías, Valores y Seguros.

Con fecha 25 de septiembre del 2020, la resolución $\mathrm{N}^{\circ}$ SCVS-INC-DNCDN-2020-0013, publicada en la Edición Especial del Registro Oficial $N^{\circ} 1076$ de la misma fecha, expide las NORMAS ECUATORIANAS DEL BUEN GOBIERNO CORPORATIVO, en materia de normas, principios, derechos de los accionistas y trato equitativo, Junta general y asamblea de accionistas, el Directorio, gobierno familiar, arquitectura de control, transparencia e información financiera y no financiera y las medidas para mitigar la corrupción empresarial.

De acuerdo con la resolución $\mathrm{N}^{\circ}$ SCVS-INC-DNCDN-2020-0013 Resolución N SCVSINC-DNCDN-2020-0017 reformas al reglamento para la recepción, sustanciación y trámite de denuncias. publicada en el registro oficial No. 330 del 16 de noviembre de 2020. Por su parte con fecha 28 de diciembre del 2020 , se expide el reglamento para la calificación y registro de compañías especializadas en valoración de activos intangibles, publicado en el registro oficial N 395 del 22 de febrero de 2021.

Por su parte con resolución SCVS-INC-DNCDN-2021-0002 (Segundo Suplemento del Registro Oficial 411, de fecha 16 de marzo del 2021, expide las normas de prevención de lavado de activos, financiamiento del terrorismo y otros delitos, la presente normativa regula fundamentalmente las políticas, procedimientos y los mecanismos para prevenir el lavado de activos, el financiamiento del terrorismo y otros delitos, que deberán observar los sujetos obligados o compañías reguladas por la Superintendencia de Compañías, Valores y Seguros, que se dediquen a las actividades establecidas en el artículo 5 de la Ley Orgánica de Prevención, Detección y Erradicación del Delito de Lavado de Activos y del Financiamiento de Delitos.

Siguiendo la misma línea tenemos, que se dispone por parte del organismo de control la prórroga del plazo previsto para la presentación de la documentación requerida en los artículos 20 y 23 de la Ley de Compañías, en concordancia con el artículo 25 del mismo cuerpo legal; en los numerales 2, 3 ,4 y 5 del artículo 5 Sección II, Capítulo II, Título IV, Libro II y el artículo 1 del Capítulo II de la Codificación de Resoluciones Monetarias, Financieras, de Valores y Seguros, hasta el 31 de mayo de 2021, según resolución de fecha 23 de abril del 2021, resolución $\mathrm{N}^{\circ}$ SCVS-INPAI-2021-0006, referente a la presentación con respecto a los estados financieros, nomina de socios y/o accionistas, nomina de administradores, estado de flujo del efectivo, estado de cambios en el patrimonio, notas a los estados financieros, informe de gerencia, informe de comisarion informe de auditoria externa y acta de junta general.

Por ultimo la resolución $\mathrm{N}^{\circ}$ SCVS-INPAI-2021-0004, de fecha 23 de abril del 2021, dispone la suspensión de los plazos y términos previstos para la presentación y 
tramitación de denuncias, reclamos impugnaciones y en general de todo proceso administrativo de competencia de la Superintendencia de Compañías, Valores y Seguros, así como para los procesos que se encuentren discurriendo en este ente de control, desde el 24 de abril hasta el 20 de mayo de 2021 inclusive.

\section{Conclusiones.}

A continuación, se exponen las principales conclusiones, integrando los resultados de los cuestionarios, y la técnica de la observación:

- Del análisis de los gráficos $\mathrm{N}^{\circ} 1$ y 2 se desprende que las microempresas con el $40,76 \%$, en nuestro estudio ocupan un papel protagónico las microempresas qque son de propiedad y administración de los propios empresarios, donde a su vez trabajan ellos mismos o con sus familiares, algunas se encuentran dentro de la economía informal, sirven para la supervivencia y no poseen capital operativo y por otra encontramos las de expansión que generan excedentes para mantener la producción, pero no hay actualmente hay muy poco crecimiento, luego estan las de mayor utilización en el Ecuador, responsabilidad limitada con el 25,05\%, y con el 23,57 las sociedad anónima, y la que viene creciendo rápidamente las sociedades simplificadas por acciones, de allí la importancia de la constitución juridca que representa el $52.02 \%$, mediante la web la Superintendencia de Compañías, fomenta y forma compañías online, de forma rápida y simple y con un menor costo, para dinamizar y reactivar la economía.

- $\mathrm{Al}$ articular los gráficos $\mathrm{N}^{\circ} 3$ y 4 se sostiene que la compra de mascarillas, guantes y otros con el $29,03 \%$ y la alta probabilidad de contagio en el $29,97 \%$, dan como resultado el $59 \%$ de problemas sociales, empresariales y psicológicos en la pandemia, de allí que es importante hacer un exhortación a considerar los aspectos de negocio, sumado a los psicológicos y sociales de la pandemia para disminuir las vulnerabilidades y el riesgo de desastre frente al COVID 19., que trajo consigo la perdida de cartera de clientes y ventas en el 23,69\% y la perdida de rentabilidad de las empresas en el orden del 12,27\%, lo que implica valores negativos.

- El analisis indico conforme grafico $\mathrm{N}^{\circ} 6$ que en un 26,54\% la inyección de capital a través de préstamos, es decir inversiones en forma de efectivo, acciones o activos que permitan tener flujo de efectivo para cubrir las obligaciones a corto plazo e impulsar mediante la creatividad e innovación nuevos productos, servicios, diseños, diversificación y comercialización, es por ello que en el $14,52 \%$ los ejecutivos empresariales sostienen que también es importante la actualización técnologica de equipos en forma integral, con el objetivo de actualizar los equipos y sistemas considerados obsoletos, utilizando métodos y equipos con técnicas y tecnología innovadoras y avanzadas para ser sustentables y sostenibles.

- En el grafico $\mathrm{N}^{\circ} 6$, es visible que el mayor problema que tienen las Mipymes han sido la baja en sus ventas en un 51,94\%, seguido de una escasez de materia prima, lo cual son problemas que se afrontan debido a que la economía mundial se ha 
visto menguada por la pandemia, ya que se han tomado mayor importancia al sector de la salud y alimentos, debido a que se ha tratado de cubrir las necesidades básicas de la población.

- La importancia de las micro, pequeñas y medianas empresas (Mipymes) en el mercado local, regional y del país, hoy por hoy constituyen la mayor fuerza económica del Ecuador. La globalización y la innovación que viene de la mano con la tecnología, imponen enormes retos a las empresas para crecer y afrontar con éxito nuevos retos y afirmar su permanencia en los mercados, para ello será importante una búsqueda continua de ser productivos, para cumplir con su misión, visión, objetivos y valores empresariales, desarrollando su potencial humano, logrando efectividad, competitividad o internacionalización de sus bienes y servicios, así como mantener su estabilidad y equilibrio para sobrevivir en el futuro. Las Mipymes se han revalorizado en el mundo por su rol en la economía, y para la sociedad en específico, por su potencial en generar empleabilidad, por su aporte con el pago de tributos e impuestos a la balanza comercial, por contribuir a reducir la concentración de poder económico y por su participación en el desarrollo y crecimiento del país.

- Se determinó que la Ley Orgánica de Apoyo Humanitario dió respuesta a la crisis de salud del COVID-19 busca establecer medidas de apoyo humanitario para hacer frente a las consecuencias de la crisis de salud provocada por la pandemia. Esta ayudando a promover la reactivación económica y productiva del Ecuador, con especial énfasis en los seres humanos, la contención y reactivación de las familias, las empresas y la economía, y el mantenimiento de las condiciones laborales.

- Se hace necesario reformar la resolución N SCVS-INC-DNCDN-2016-011 de 21 de septiembre de 2016, publicada en el Registro Oficial No. 879 de 11 de noviembre de 2016, que contenga un reglamento sobre Auditoría Externa, en donde las compañías nacionales de economía mixta, anónimas y sociedades por acciones simplificadas con participación de personas jurídicas de derecho público o de derecho privado con finalidad social o pública, cuyos activos excedan de cien mil dólares de los Estados Unidos de América (US\$ 100.000,00), deban necesariamente tener auditoria externa, de tal manera que permitan examinar las prácticas financieras de los negocios para asegurar que este siga los principios de las Normas Internacionales de Contabilidad y Normas Internacionales de Información Financiera, aceptados y detectar problemas potenciales o reales como errores, fraudes y tendencias específicas de rendimiento en una empresa.

- Es conveniente que las compañias adopten de forma seria y responsable las "Normas Ecuatorianas para el Buen Gobierno Corporativo", cuya implementación en todo el sector empresarial es una aspiración válida, porque este sistema de organización y gestión promueve la transparencia de información, evita el conflicto y pugna de intereses entre socios accionistas mayoritarios y minoritarios, fortalece las relaciones del nivel directivo empresarial, estableciendo procesos normados para la sucesión de los directivos, da respuestas sólidas ante situaciones 
de contingencia, entre otras múltiples ventajas que dicho sistema proporciona a las compañías que lo apliquen.

\section{Referencias bibliográficas.}

Código Civil de la República del Ecuador. (2005). Obtenido de https://www.correosdelecuador.gob.ec/wpcontent/uploads/downloads/2017/08/codigo-civil.pdf

Constitución de la República del Ecuador. (2008). Obtenido de http://goberguayas.gob.ec/pdf/INFORMACIONLEGAL/NORMASDECREACI ON/Norma-Constitucional.pdf

Fondo Monetario Internacional (FMI). 2020c. "La política fiscal en tiempos de pandemia: ¿Cómo le ha ido a América Latina y el Caribe?” Perspectivas económicas: Las Américas. Estudio de referencia No. 3, Washington, DC, octubre

Hurtado de Barrera, J. (2010). Guía para la comprensión holística de la ciencia, Tercera Edición, Fundación Sypal: Caracas-Venezuela.

La Comisión Económica para América Latina - CEPAL (2021), obtenido de: https://www.cepal.org/es/euromipyme/mipymes-covid-19

Ley de Compañías de la República del Ecuador. (2014). Obtenido de https://www.supercias.gob.ec/bd_supercias/descargas/lotaip/a2/Ley-Cias.pdf

Ley Orgánica de apoyo humanitario (2020). Gaceta oficial № 229, del 22 de junio del 2020.

Organización Mundial de la Salud -OMS (2021) Obtenido de https://www.ilo.org/wcmsp5/groups/public/---americas/---ro- lima/documents /publication/wcms_763188.pdf

SENPLADES. (2017). Plan Nacional de Desarrollo Toda una Vida 2017-2021. Quito: SENPLADES.

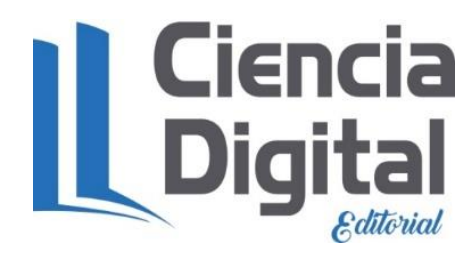




\section{PARA CITAR EL ARTÍCULO INDEXADO.}

Becerra Molina, E., Astudillo Arias, P., \& Jaramillo Calle, Y. (2021). Alcance de la información societaria, durante la pandemia del COVID-19. AlfaPublicaciones, 3(3), 120-138. https://doi.org/10.33262/ap.v3i3.72

\section{¿Ciencia}

El artículo que se publica es de exclusiva responsabilidad de los autores y no necesariamente reflejan el pensamiento de la Revista Alfa Publicaciones.

El artículo queda en propiedad de la revista y, por tanto, su publicación parcial y/o total en otro medio tiene que ser autorizado por el director de la Revista Alfa Publicaciones.
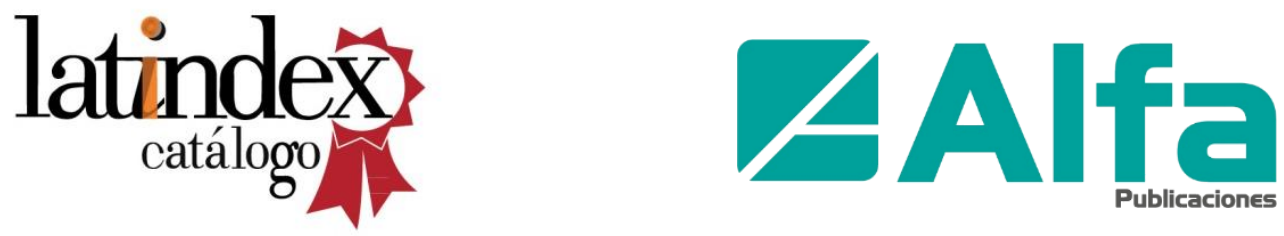